\title{
Convergence and Isotopy Type for Graphs of Finite Total Curvature
}

\author{
Elizabeth Denne and John M. Sullivan

\begin{abstract}
Generalizing Milnor's result that an FTC (finite total curvature) knot has an isotopic inscribed polygon, we show that any two nearby knotted FTC graphs are isotopic by a small isotopy. We also show how to obtain sharper constants when the starting curve is smooth. We apply our main theorem to prove a limiting result for essential subarcs of a knot.
\end{abstract}

Keywords. Knots, knotted graphs, isotopy, convergence.

\section{Introduction}

A tame knot or link type is one that can be represented by a polygonal space curve. It is clear that the corners of a polygon can be rounded off, so that a tame link can also be represented by a smooth, even $C^{\infty}$, curve. Conversely, given a $C^{2}$ curve, it is relatively easy to find an isotopic inscribed polygon. With less smoothness, the arguments become more intricate but are by now standard. Crowell and Fox [CF63, App. I] gave a detailed proof for $C^{1}$ curves, while Milnor [Mil50] introduced the class of finite total curvature (FTC) curves, and showed they also have isotopic inscribed polygons.

Thus any embedded FTC curve represents a tame link type; but tame links (even the unknot) can of course also be represented by curves of infinite total curvature or even infinite length. However, our work in geometric knot theory has suggested to us that FTC links are a very useful class, which might be considered as "geometrically tame". (See [Sul08], in this volume, for a survey on FTC curves. We show for instance that this is the natural class for standard results like Schur's comparison theorem.)

Here, with a view towards convergence results in geometric knot theory, we examine the question of how close two space curves must be in order to be (ambient) isotopic. Of course, the phenomenon of local knotting means that our notion of distance between space curves must control tangent directions as well as position. (Otherwise, within any distance $\varepsilon$ of any knot there would be infinitely many composite knots-with extra summands contained in $\varepsilon$-balls_and even certain prime satellites of the original knot.) We 
also show that, when the initial curves are close enough, the ambient isotopy can be made arbitrarily small.

The arguments of [CF63, Mil50] not only produce one isotopic inscribed polygon, but in fact show that all sufficiently fine inscribed polygons are isotopic to a given $C^{1}$ or FTC curve. We recover this result, since finely inscribed polygons are nearby in our sense (with one caveat, discussed later).

We prove three versions of our theorem. The first is restricted curves of positive thickness, that is, to $C^{1,1}$ links, but it allows us to get nearly optimal bounds (in terms of the thickness) for how close the two curves must be before they are guaranteed to be isotopic. The second version, our main theorem, applies not just to links but to arbitrary knotted FTC graphs; it gives us control over the distance points are moved by the isotopy. The final version applies to $C^{1}$ links and allows us to prove that the isotopy is small even in a stronger $C^{1}$ sense.

As an application of the main theorem, we consider the notion of essential arcs and secants of a knot, defined [DDS06] in terms of certain knotted $\Theta$-graphs. We show that essential secants remain essential in limits, which is important for results [Den04] on quadrisecants.

\section{Definitions}

Definition. A (knotted) rectifiable graph is a (multi-)graph-of fixed finite combinatorial type-embedded in space such that each edge is a rectifiable arc. An FTC graph is a rectifiable graph where each arc has finite total curvature.

We note that Taniyama [Tan98] has considered minimization of the total curvature of knotted graphs, including analogs of the Fáry/Milnor theorem.

For an introduction to the theory of knotted graphs, see [Kau89]: in particular, we are interested in graphs with what Kauffman calls topological (rather than rigid) vertices. The $k$ edges incident to a vertex $v$ can be braided arbitrarily near $v$ without affecting the knot type of the graph. We do not require our graphs to be connected, so our results apply to links as well as knots.

As mentioned above, in order to conclude that nearby knots are isotopic, we need to use a notion of distance that controls tangent vectors. A standard notion would be, for instance, $C^{1}$-convergence of $C^{1}$ curves. Our goal, however, is to consider curves which need not be $C^{1}$. Recalling that a rectifiable curve has a well-defined tangent vector almost everywhere, we can make the following definition.

Definition. Given two rectifiable embeddings $\Gamma$ and $\Gamma^{\prime}$ of the same combinatorial graph, we say they are $(\delta, \theta)$-close if there exists a homeomorphism between them such that corresponding points are within distance $\delta$ of each other, and corresponding tangent vectors are within angle $\theta$ of each other almost everywhere.

Remember [Sul08] that any curve or graph $\Gamma$ of finite total curvature has welldefined one-sided tangent vectors everywhere; these are equal and opposite except at 
countably many corners of $\Gamma$ (including of course the vertices of the graph). In the definition of $(\delta, \theta)$-close it would be equivalent-in the case of FTC or piecewise $C^{1}$ graphs- to require that the one-sided tangent vectors be everywhere within angle $\theta$.

\section{Isotopy for thick knots}

Our first isotopy result looks at thick curves (that is, embedded $C^{1,1}$ curves). Here we can get close to optimal bounds on the $\delta$ and $\theta$ needed to conclude that nearby curves are isotopic.

The thickness $\tau(K)$ of a space curve $K$ is defined [GM99] to be twice the infimal radius of circles through any three distinct points of $K$. A link is $C^{1,1}$ (that is, $C^{1}$ with Lipschitz tangent vector, or equivalently with a weak curvature bound) if and only if it has positive thickness [CKS02]. Of course when $K$ is $C^{1}$, we can define normal tubes around $K$, and then $\tau(K)$ is the supremal diameter of such a tube that remains embedded. (We note that in the existing literature thickness is sometimes defined to be the radius rather than diameter of this thick tube.) Points inside the tube have a unique nearest neighbor on $K$ : the tube's radius is also the reach of $K$ in the sense of Federer [Fed59].

Fix a link $K$ of thickness $\tau>0$. Any other link which follows $K$ once around within its thick tube, transverse to the normal disks, will be isotopic to $K$. This might seem to say that any link $(\tau / 2, \pi / 2)$-close to $K$ is isotopic, but this would only work if we knew the correspondence used in the definition of closeness were the closest-point projection to $K$. We do see that $(\delta, \theta)$-closeness is no guarantee of isotopy if $\theta>\pi / 2$ (allowing local knotting) or if $\delta>\tau / 2$ (allowing global strand passage). So the following proposition (which extends Lemma 6.1 from [DDS06], with essentially the same proof) is certainly close to optimal.

(We note, however, that $\delta>\tau / 2$ only allows global strand passage in the case where the thickness of $K$ is controlled by self-distance rather than curvature. This caveat explains why we can later get some analogs of the following proposition even for curves whose thickness is zero because they have corners.)

Proposition 3.1. Fix a $C^{1,1}$ link $K$ of thickness $\tau>0$. For any $\delta<\tau / 4$, set $\theta:=$ $\pi / 2-2 \arcsin (2 \delta / \tau)$. Then any (rectifiable) link $K^{\prime}$ which is $(\delta, \theta)$-close to $K$ is ambient isotopic; the isotopy can be chosen to move no point a distance more than $\delta$.

Proof. For simplicity, rescale so that $\tau=1$, meaning the curvature of $K$ is bounded by 2 . Suppose $p^{\prime}$ and $p$ are corresponding points on $K$ and $K^{\prime}$, so $\left|p^{\prime}-p\right|<\delta$. Let $p_{0}$ be the (unique) closest point on $K$ to $p^{\prime}$. Then of course $\left|p^{\prime}-p_{0}\right| \leq\left|p^{\prime}-p\right|<\delta$, so $\left|p-p_{0}\right|<2 \delta$. Standard results on the geometry of thick curves [DDS06, Lem. 3.1] show that the arclength of $K$ between $p$ and $p_{0}$ is at most $\arcsin (2 \delta)$, so by the curvature bound, the angle between the tangents $T_{p}$ and $T_{p_{0}}$ at these two points is at most $2 \arcsin (2 \delta)$. The definition of $(\delta, \theta)$-close means that the tangent vector (if any) to $K^{\prime}$ at $p^{\prime}$ makes angle less than $\theta$ with $T_{p}$, so by definition of $\theta$ it makes angle less than $\pi / 2$ with $T_{p_{0}}$.

This shows that $K^{\prime}$ is transverse to the foliation of the thick tube around $K$ by normal disks. We now construct the isotopy from $K^{\prime}$ to $K$ as the union of isotopies in 
these disks; on each disk we move $p^{\prime}$ to $p_{0}$, coning this outwards to the fixed boundary. No point in the disk moves further than $p^{\prime}$ does, and this is less than $\delta$.

\section{Isotopy for graphs of finite total curvature}

When generalizing our isotopy results to knotted graphs, we must allow corners at the vertices of the graph, so it is natural to consider FTC graphs with possibly additional corners along the arcs. Our main theorem will again show that sufficiently close curves are isotopic.

Any curve with a corner has zero thickness $\tau=0$, so it is perhaps surprising that an analog of Proposition 3.1 still holds. Perhaps the maximum possible value of $\delta$ here could be taken as a different notion thickness of the curve; we do not explore this idea, but content ourselves with merely proving the existence of some positive $\delta$.

A preliminary combing lemma allows the main theorem to treat vertices on knotted graphs as well as sharp corners along a single strand. It was inspired by Alexander and Bishop's correction [AB98] to Milnor's treatment of corners of angle $\pi$. This case-where two or more strands leave a corner in the same direction, allowing infinite winding or braiding - is the most difficult, and seems to be the obstacle to creating an isotopy which is $(\delta, \theta)$-small in the sense described in Section 7 . But this case does not need to be treated separately in our proof.

This combing lemma is an example of the "Alexander trick" (compare [Thu97, Prob. 3.2.10]). In general the trick shows that any homeomorphism of the unit $d$-ball which fixes the boundary is isotopic to the identity.

Lemma 4.1. Let $B$ be a round ball centered at $p$, and suppose graphs $\Gamma$ and $\Gamma^{\prime}$ each consist of $n$ labeled arcs starting at $p$ and proceeding out to $\partial B$ transverse to the nested spheres around $p$. Then $\Gamma$ and $\Gamma^{\prime}$ are ambient isotopic. In fact, any isotopy of $\partial B$ which takes the $n$ points of $\Gamma$ to those of $\Gamma^{\prime}$ can be extended to an isotopy of the whole ball.

Proof. It suffices to show that $\Gamma$ is isotopic to a configuration with $n$ straight radial segments to the same $n$ points on $\partial B$. For then the same is true of $\Gamma^{\prime}$, and any two straight configurations are clearly isotopic (for instance by extending radially any given boundary isotopy).

To straighten $\Gamma$, we comb the $n$ strands inwards from $\partial B$, which we identify with the unit sphere. For $0<\lambda \leq 1$, let $p_{i}(\lambda)$ be the $n$ points of intersection of $\Gamma$ with the sphere $\lambda \partial B$. Start with $f_{1}$ being the identity map, and define a family of maps $f_{\lambda}$ from the unit sphere to itself, continuous in $\lambda$, such that $f_{\lambda}$ takes $p_{i}(1)$ to $p_{i}(\lambda) / \lambda$ for each $i$. The combing isotopy, at each time $t$, maps each concentric sphere $\lambda \partial B$ to itself, using the map $f_{\lambda+(1-\lambda) t} \circ f_{\lambda}^{-1}$. Thus at time $t$ on the sphere of size $\lambda$, we see the same picture that was initially on the sphere of size $\lambda+(1-\lambda) t$; see Figure 1 It follows that at time $t=1$ the strands are all straight.

As we have mentioned, if two (or more) strands of $\Gamma$ leave $p$ with a common tangent, then they might twist (or braid) around each other infinitely often. But outside any sphere 

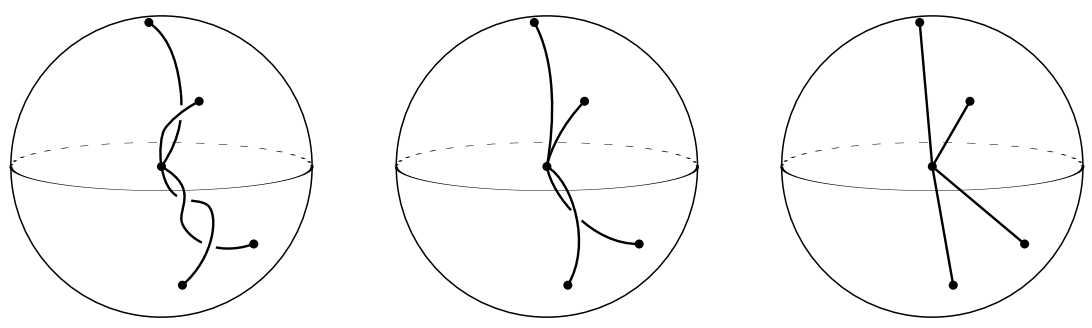

FIGURE 1. The isotopy of Lemma 4.1 straightens strands in a ball by combing them inwards. Only the outer half of the time 0 pattern (left) is still seen at time $\frac{1}{2}$ (center); at time 1 (right) we have straight radii.

$\lambda \partial B$ they can twist only finitely many times, explaining why the isotopy constructed in the lemma has no trouble combing this out.

Theorem 4.2. Suppose $\Gamma$ is a knotted graph of finite total curvature and $\varepsilon>0$ is given. Then there exists $\delta>0$ such that any (rectifiable) graph $\Gamma^{\prime}$ which is $(\delta, \pi / 8)$-close to $\Gamma$ is ambient isotopic to $\Gamma$, via an isotopy which moves no point by more than $\varepsilon$.

Proof. We begin by selecting a finite number of points $p_{j}$ on $\Gamma$ (including all its vertices) such that these points divide $\Gamma$ into $\operatorname{arcs} \alpha_{k}$ each of total curvature less than $\pi / 8$. (Note that any corner in $\Gamma$ of turning angle at least $\pi / 8$ must be included among the $p_{j}$.)

Let $r_{1}$ be the minimum distance between any two arcs $\alpha_{k}$ which are not incident to a common $p_{j}$ (or the minimum distance between points $p_{j}$, if this is smaller). Set $r_{2}:=\min \left(r_{1} / 2, \varepsilon / 2\right)$. Consider disjoint open balls $B_{j}$ of radius $r_{2}$ centered at the $p_{j}$. Each arc $\alpha_{k}$ leaving $p_{j}$ proceeds monotonically outwards to the boundary of $B_{j}$ (since its curvature is too small to double back). Also, $B_{j}$ contains no other arcs (since $r_{2}$ was chosen small enough); indeed no other arcs come within distance $r_{2}$ of $B_{j}$.

Note that $\Gamma \backslash \bigcup B_{j}$ is a compact union of disjoint arcs $\beta_{k} \subset \alpha_{k}$. Let $r_{3}$ be the minimum distance between any two of these arcs $\beta_{k}$. Considering endpoints of various $\beta_{k}$ on the boundary of a single $B_{j}$, we note that $r_{3} \leq 2 r_{2}<\varepsilon$.

For each $\beta_{k}$, we construct a tube $T_{k}$ around it as follows. Suppose $p_{j}$ and $p_{j^{\prime}}$ are the two endpoints of $\alpha_{k} \supset \beta_{k}$. Foliate $\mathbb{R}^{3} \backslash\left(B_{j} \cup B_{j^{\prime}}\right)$ by spheres of radius at least $r_{2}$ (and one plane, bisecting $\overline{p_{j} p_{j^{\prime}}}$ ) in the obvious smooth way. (Their signed curvatures vary

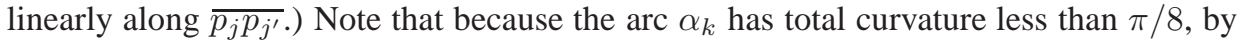
[Sul08, Lem. 2.3] it is contained in a spindle of revolution from $p$ to $p^{\prime}$, of curvature $\pi / 4$. Within this spindle, the normal vectors to the foliating spheres stay within angle $\pi / 8$ of $\overline{p_{j} p_{j^{\prime}}}$, as do the (one-sided) tangent vectors to $\alpha_{k}$. Thus $\beta_{k}$ is within angle $\pi / 4$ of being normal to the foliation; in particular it is transverse. Finally, we set $r_{4}:=r_{3} / 6$ and, for each point $q \in \beta_{k}$, we consider the foliating sphere through $q$ and in particular its intersection with $B_{r_{4}}(q)$, a (slightly curved) disk $D_{q}$. The tubular neighborhood $T_{k}$ is the union of these disks over all $q \in \beta_{k}$. Since $r_{4}<r_{2} / 3$, the normal vector along each $D_{q}$ stays within angle $2 \arcsin \frac{1}{6}<\pi / 8$ of that at its center $q$, hence within $\pi / 4$ of $\overline{p_{j} p_{j^{\prime}}}$. 


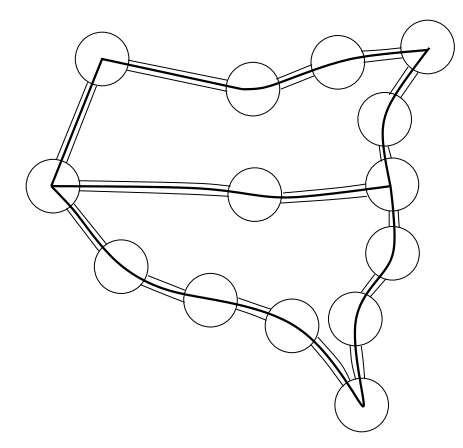

FIGURE 2. An FTC graph has a neighborhood consisting of balls and tubes, inside of which we perform our isotopy to any nearby graph.

We have thus found a neighborhood $N$ of $\Gamma$ which, as shown in Figure 2 is foliated by round spheres near the $p_{j}$ and almost flat disks along the $\beta_{k}$; the graph $\Gamma$ is transverse to this foliation $F$.

Now take $\delta:=r_{4} / 3<r_{2} / 9$ and consider some $\Gamma^{\prime}$ which is $(\delta, \pi / 8)$-close to $\Gamma$. We will see that $\Gamma^{\prime}$ lies within our neighborhood $N$ and is also transverse to the foliation $F$, except perhaps near the $p_{j}$. To find an ambient isotopy from $\Gamma^{\prime}$ to $\Gamma$, we proceed in two stages.

First we find the points $p_{j}^{\prime} \in \Gamma^{\prime}$ corresponding to $p_{j} \in \Gamma$, and construct an isotopy $I$ supported in the $B_{j}$ as follows: the map moves each point along a straight line; $p_{j}^{\prime}$ moves to $p_{j}$, each boundary point $q \in \partial B_{j}$ is fixed, and the map is linear on each segment $\overline{p_{j} q}$. Because $\Gamma^{\prime}$ was $(\delta, \pi / 8)-$ close to $\Gamma$, and because $p_{j}^{\prime}$ was so close to $p_{j}$ compared to the radius $r_{2}$ of $B_{j}$, the resulting $\Gamma^{\prime \prime}:=I_{1}\left(\Gamma^{\prime}\right)$ at time 1 has radial strands within each $B_{j}$, so we will be able to use the combing lemma.

In fact, we claim that $\Gamma^{\prime \prime}$ is contained in $N$ and transverse to the foliation $F$. The original correspondence between $\Gamma^{\prime}$ and $\Gamma$ (showing they were $(\delta, \pi / 8)$-close) did not pair points in the same leaf of the foliation. But outside the $B_{j}$, for each point $q^{\prime}$ on the arc of $\Gamma^{\prime \prime}=\Gamma^{\prime}$ corresponding to $\beta_{k}$, consider the leaf of the foliation it lies in and the unique point $q_{0} \in \beta_{k}$ on the same leaf. The distance $\left|q^{\prime}-q_{0}\right|$ might be slightly bigger than the distance $\left|q^{\prime}-q\right|<\delta$ in the original pairing. However, since the angles of the curves and the foliation are so well controlled, it is certainly well less than $2 \delta<r_{4}$; the tangent directions at $q$ and $q_{0}$ are certainly within $\pi / 8$ of each other. That is, even under the new correspondance $\left(q^{\prime}\right.$ to $\left.q_{0}\right)$ these $\operatorname{arcs}$ of $\Gamma^{\prime}$ and $\Gamma$ are $(2 \delta, \pi / 4)$-close. This proves the claim.

Second, we construct an isotopy $J$ which is supported in $N$ and which preserves each leaf of the foliation $F$. On the disks in the tubes $T_{k}$ this is easy: we have to move one given point in the disk to the center, and can choose to do this in a continuous way. On the boundary $\partial B_{j}$ we define $J$ to match these motions in the disks and fix the rest of the sphere. Finally, we use Lemma4.1 to fill in the isotopy $J$ in the interior of the balls. 
By construction, the overall isotopy $I$ followed by $J$ moves points less than $\varepsilon$, as desired: within each ball $B_{j}$ we have little control over the details but certainly each point moves less than the diameter $2 r_{2}<\varepsilon$; within $T_{k}$ each point moves less than $r_{4} \ll \varepsilon$.

Even though we have emphasized the class of FTC curves, we note that a very similar proof could be given for the case when $\Gamma$ is piecewise $C^{1}$. We would simply choose the points $p_{j}$ such that the intervening arcs $\alpha_{k}$, while potentially of infinite total curvature, had tangent vectors staying within angle $\pi / 8$ of the vector between their endpoints. Compare the beginning of the proof of Proposition 7.3 below.

As we noted in the introduction, we can recover the following result: given any FTC (or $C^{1}$ ) link, any sufficiently fine inscribed polygon is isotopic. When the link is $C^{1}$, this is an immediate corollary, sine the polygon will be $(\delta, \theta)$-close. For an FTC link $K$ with corners, even very finely inscribed polygons will typically cut those corners and thus deviate by more than angle $\pi / 8$ from the tangent directions of $K$. But, using Lemma 4.1 near those sharp corners, we see immediately that the polygon is isotopic to one that does use the corner as a vertex, and thus to $K$.

\section{Tame and locally flat links and graphs}

Remember that a tame link is one isotopic to a polygon. Tame links clearly satisfy the following local condition.

Definition. A link $K$ is locally flat if each point $p \in K$ has a neighborhood $U$ such that $(U, U \cap K)$ is an unknotted ball-arc pair. (Unknotted means that the pair is homeomorphic to a round ball with its diameter.)

We can generalize the definitions to knotted graphs.

Definition. A knotted graph $\Gamma$ is tame if it is isotopic to a polygonal embedding; it is locally flat if each point $p \in \Gamma$ has a neighborhood $U$ such that $(U, U \cap \Gamma)$ is homeomorphic to a standard model. At a $k$-fold vertex this standard model is a round ball with $k$ of its radii; along an arc of $\Gamma$ it is an unknotted ball-arc pair.

Again, tame graphs are clearly locally flat. In the mid-1950s, Bing [Bin54 and Moise [Moi54] independently proved the much more difficult converse: any locally flat graph is tame.

Our main theorem showed that an FTC graph is isotopic to any other nearby graph. This implies that FTC graphs are tame: as long as we include sharp corners among the polygon vertices then sufficiently fine inscribed polygons will be $(\delta, \pi / 8)$-close.

Here we note that it is much easier to prove directly that FTC graphs are locally flat.

Proposition 5.1. Graphs of finite total curvature are locally flat.

Proof. Let $p_{0} \in \Gamma$ be any point along a graph of finite total curvature. Repeat the construction at the beginning of the proof of Theorem 4.2 but include $p_{0}$ among the $p_{j}$. This gives a ball $B_{0}$ around $p_{0}$ containing only $k$ radial arcs of $\Gamma$. By Lemma 4.1 this ball is homeomorphic to any other such ball, in particular to the standard model. 


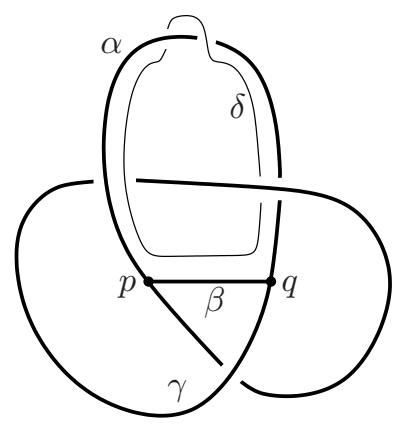

FIGURE 3. In this knotted $\Theta$-graph the ordered triple $(\alpha, \beta, \gamma)$ is essential. The curve $\delta$ is the parallel to $\alpha \cup \beta$ having linking number zero with $\alpha \cup \gamma$. The fact that it is homotopically nontrivial in the knot complement $\mathbb{R}^{3} \backslash(\alpha \cup \gamma)$ is the obstruction to the existence of a spanning disk. In this illustration, $\beta$ is the straight segment $\overline{p q}$, so we equally say that the arc $\alpha$ of the $\operatorname{knot} \alpha \cup \gamma$ is essential.

\section{Applications to essential arcs}

One strand of work in geometric knot theory attempts to show that knotted curves are geometrically more complex than unknots. One of the earliest results in this direction is the Fáry/Milnor theorem, which says a knotted curve has more than twice the total curvature of a round circle. Interesting measures of geometric complexity include ropelength [CKS02] and distortion [KS97].

We have recently obtained new lower bounds for both ropelength [DDS06] and distortion [DS04] of knotted curves using the notion of essential arcs. Generically, a knot $K$ together with one of its chords $\overline{p q}$ forms a $\Theta$-graph in space; being essential is a topological feature of this knotted graph. The following definition, introduced in [Den04, DDS06] and illustrated in Figure 3, is an extension of ideas of Kuperberg [Kup94].

Definition. Suppose $\alpha, \beta$ and $\gamma$ are three disjoint simple arcs from $p$ to $q$, forming a knotted $\Theta$-graph in $\mathbb{R}^{3}$. We say that $(\alpha, \beta, \gamma)$ is inessential if there is a disk $D$ bounded by the knot $\alpha \cup \beta$ and having no interior intersections with the knot $\alpha \cup \gamma$. (We allow self-intersections of $D$, and interior intersections with $\beta$; the latter are necessary if $\alpha \cup \beta$ is knotted.)

Now suppose $p$ and $q$ are two points along a knot $K$, dividing it into complementary subarcs $\gamma_{p q}$ and $\gamma_{q p}$. We say $\gamma_{p q}$ is essential in $K$ if for every $\varepsilon>0$ there exists some $\varepsilon$-perturbation $S$ of $\overline{p q}$ (with endpoints fixed) such that $K \cup S$ is an embedded $\Theta$-graph in which $\left(\gamma_{p q}, S, \gamma_{q p}\right)$ is essential.

Allowing the $\varepsilon$-perturbation ensures that the set of essential arcs is closed in the set of all subarcs of $K$; it also lets us handle the case when $S$ intersects $K$. We could allow the perturbation only in case of such intersections; applying an argument like Lemma 4.1 in the case of an embedded $\Theta$ (no intersections) shows this definition would be equivalent, 
at least when the knot $K$ is FTC. We require only that the perturbation be small in a pointwise sense. Thus $S$ could be locally knotted, but since the disk in the definition can intersect $\beta=S$ this is irrelevant.

This definition of essential in terms of $\Theta$-graphs was what led us to consider knotted graphs and to look for a result like Theorem 4.2. We now apply that theorem to show that essential arcs are preserved in limits. (In [DDS06, Prop. 6.2] we gave a similar result, but since we did not yet have Theorem 4.2 we had to restrict to $C^{1,1}$ knots only. The proof below does closely follow the proof there, Theorem 4.2 being the main new ingredient.)

Proposition 6.1. Suppose a sequence of rectifiable knots $K_{i}$ converge to an FTC limit $K$ in the sense that $K_{i}$ is $\left(\delta_{i}, \pi / 10\right)$-close to $K$ with $\delta_{i} \rightarrow 0$. Suppose that the $K_{i}$ have essential subarcs $\gamma_{p_{i} q_{i}}$ with $p_{i} \rightarrow p$ and $q_{i} \rightarrow q$. Then $\gamma_{p q}$ is an essential subarc of $K$.

Proof. We can reduce to the case $p_{i}=p, q_{i}=q$ (but with $\pi / 10$ above replaced by $\pi / 8$ ) by appyling euclidean similarities (approaching the identity) to the $K_{i}$.

Given any $\epsilon>0$, we prove there is an $2 \epsilon$-perturbation $S$ of $\overline{p q}$ for which $\left(\gamma_{p q}, S, \gamma_{q p}\right)$ is essential. Then by definition $\gamma_{p q}$ is essential.

We want to find an ambient isotopy $I$ from some $K_{i}$ to $K$ which moves points less than $\varepsilon$. So apply Theorem 4.2 to $K$ to determine $\delta>0$ and then pick $i$ large enough so that the knot $K_{i}$ is $(\delta, \pi / 8)$-close to $K$. The ambient isotopy $I$ guaranteed by Theorem 4.2 , with $K=I\left(K_{i}\right)$, moves points less than $\varepsilon$, as desired.

Since $\gamma_{p_{i} q_{i}} \subset K_{i}$ is essential, by definition, we can find an $\epsilon$-perturbation $S_{i}$ of $\overline{p_{i} q_{i}}$ such that $\left(\gamma_{p_{i} q_{i}}, S_{i}, \gamma_{q_{i}, p_{i}}\right)$ is essential. Setting $S:=I\left(S_{i}\right)$, this is the desired $2 \epsilon$-perturbation of $\overline{p q}$. By definition, the $\Theta$-graph $K_{i} \cup S_{i}$ is isotopic via $I$ to $K \cup S$, so in the latter $\left(\gamma_{p q}, S, \gamma_{q p}\right)$ is also essential.

\section{Small isotopies in a stronger sense}

The isotopy constructed in Proposition 3.1 is small in a pointwise sense. But we have required our curves to be close in a stronger sense. We will say that a $C^{1}$ isotopy $I$, or more precisely its time-1 map $I_{1}$, is $(\delta, \theta)$-small if $I_{1}$ moves no point distance more than $\delta$ and its derivative turns no tangent vector more than angle $\theta$. Note that under such a small isotopy, every rectifiable graph $\Gamma$ is $(\delta, \theta)$-close to its image $I_{1}(\Gamma)$. (Although we could extend the definition of "small" for certain nonsmooth isotopies, we do not pursue this idea here. It remains unclear if the ball isotopy of Lemma 4.1 could be made small in some such sense.)

Lemma 7.1. Suppose an ambient isotopy is given for $t \in[0,1]$ by $I_{t}(p)=p+t f(p)$, where $f: \mathbb{R}^{3} \rightarrow \mathbb{R}^{3}$ is smooth, bounded in norm by $\delta$, and $\lambda$-Lipschitz. Then $I$ is $(\delta, \arctan \lambda)-$ small.

Proof. Clearly each point $p \in \Gamma$ is within distance $\delta$ of $p^{\prime}:=I_{1}(p)$. If $v$ is the unit tangent vector to $\Gamma$ at $p$, then its image, the tangent vector to $\Gamma^{\prime}$ at $p^{\prime}$, is $v+\partial_{v} f$, where $\left|\partial_{v} f\right|<\lambda$ by the Lipschitz condition. This differs in angle from $v$ by at most $\arctan \lambda$. 
The isotopy we built disk-by-disk in Proposition 3.1 is not necessarily smooth, and does not necessarily satisfy the hypotheses of this lemma. In particular, since $K$ might not be piecewise $C^{2}$, its normal disks might not form a piecewise $C^{1}$ foliation, and the resulting isotopy would not even have one-sided derivatives everywhere.

So we will use a different construction, not using the thick tube. Here, as in Theorem 4.2 we abandon any attempt to get optimal constants, but the starting curve can be any $C^{1}$ curve, as in Crowell and Fox's construction [CF63, App. I] of an isotopic inscribed polygon. We begin with a lemma which captures what we feel is the essential ingredient of that argument.

Lemma 7.2. Given a $C^{1}$ link $K$ and an angle $\theta>0$, there is some $\ell>0$ such that along any subarc $\alpha$ of $K$ of length at most $\ell$, the tangent vector stays within angle $\theta$ of the chord vector $v$ connecting the endpoints of $\alpha$.

Proof. Because $K$ is compact, its continuous tangent vector is in fact uniformly continuous. Noting that $v$ is an average of the tangent vectors along $\alpha$, the result follows immediately.

This implies that the distortion (the supremal arc/chord ratio) of any $C^{1}$ curve is bounded: by the lemma the ratio approaches 1 for short arcs, so the supremum is achieved.

Proposition 7.3. Given a $C^{1}$ link $K$, we can find $\varepsilon>0$ such that the following holds. For any $\delta<\varepsilon$ and $\theta<\pi / 6$, and any $C^{1}$ link $K^{\prime}$ which is $(\delta, \theta)$-close to $K$, there is a $(2 \delta, 2 \theta)$-small ambient isotopy taking $K^{\prime}$ to $K$.

Sketch of proof. Fix $\varphi<5^{\circ}$, and find $\ell>0$ as in Lemma7.2 Now set $\tau:=\min |x-y|$, where the minimum is taken over all points $x, y \in K$ not connected by a subarc of length less than $\ell$. (Note that $\tau<\ell$.)

Along each component of $K$, pick $r \in(\tau / 50, \tau / 40)$ and place points $p_{j}$ spaced equally at arclength $r$. The resulting inscribed polygon $P$ has edgelengths in the interval $[r \cos \varphi, r]$ and is $(r \sin \varphi, \varphi)$-close to $K$, with turning angles at the $p_{j}$ less than $2 \varphi$. Rounding off the corners in a suitable fashion, we obtain a $C^{2}$ curve $L$ whose radius of curvature is never less than $5 r$, and which is $(d, \varphi)$-close to $P$ for $d \leq(\sec \varphi-1) r / 2<$ $r / 500$. We see that $L$ is $(\tau / 400,2 \varphi)$-close to $K$, and has radius of curvature at least $\tau / 10$.

We claim that the normal tube around $L$ of diameter $\tau / 5$ is embedded. This is true locally by the curvature bound. By standard results on thickness (cf. [CKS02, DDS06]) it can then only fail if there is a doubly-critical pair on $L$ at distance less than $\tau / 5$ : a pair whose chord is perpendicular to $L$ at both ends. Then there would be a nearby pair on $K$, still at distance less than $\tau / 4$. Since an arc of $L$ connecting the doubly critical pair must turn at least $\pi$, the pair on $X$ cannot be connected by an arc of length less than $\ell$. Thus we have a contradiction to the choice of $\tau$.

Now choose $\varepsilon:=\tau / 100$. For any $\delta<\varepsilon$ and $\theta<\pi / 6$, if $K^{\prime}$ is $(\delta, \theta)$-close to $K$, then it is easy to check that both are $C^{1}$, transverse sections in the tube of radius $\tau / 50$ around $L$. We construct an isotopy between them, supported in the embedded tube of radius $\tau / 10$ around $L$. On each normal disk, we move a subdisk of radius $\tau / 25$ rigidly, to move its center from $K$ to $K^{\prime}$. In the outer part of the thick tube, we extend in such a 
way that the isotopy is $C^{1}$ throughout space. The isotopy moves no points more than the distance between $K$ and $K^{\prime}$ in the normal disk. This may be somewhat more than $\delta$, since the original pairing between the two links may have been different, but is certainly less than $2 \delta$.

To check that this isotopy turns tangent vectors at most $2 \theta$, it is easiest to apply Lemma 7.1. The Lipschitz constant $\lambda$ is basically determined by needing to turn $K^{\prime}$ by approximately angle $\theta$ to match $K$. Because the normal disks get slightly closer to one another as we move off $L$ in the direction of its curvature, and since we have smoothed outside the thinner tube, the constant goes up slightly, but not enough to violate the $2 \theta$.

\section{Acknowledgments}

We extend our thanks to Stephanie Alexander, Dick Bishop and Dylan Thurston for their interest and helpful conversations.

\section{References}

[AB98] Stephanie B. Alexander and Richard L. Bishop, The Fáry-Milnor theorem in Hadamard manifolds, Proc. Amer. Math. Soc. 126:11 (1998), 3427-3436.

[Bin54] RH Bing, Locally tame sets are tame, Ann. of Math. (2) 59:1 (1954), 145-158.

[CF63] Richard H. Crowell and Ralph H. Fox, Introduction to knot theory, GTM 57, Springer, 1963.

[CKS02] Jason Cantarella, Robert B. Kusner, and John M. Sullivan, On the minimum ropelength of knots and links, Invent. Math. 150 (2002), 257-286; arXiv.org/math.GT/ 0103224.

[DDS06] Elizabeth Denne, Yuanan Diao, and John M. Sullivan, Quadrisecants give new lower bounds for the ropelength of a knot, Geometry and Topology 10 (2006), 1-26; arXiv . org/math.DG/0408026.

[Den04] Elizabeth Denne, Alternating quadrisecants of knots, Ph.D. thesis, Univ. Illinois, Urbana, 2004, arXiv.org/math.GT/0510561.

[DS04] Elizabeth Denne and John M. Sullivan, The distortion of a knotted curve, preprint, 2004, arXiv.org/math.GT/0409438.

[Fed59] Herbert Federer, Curvature measures, Trans. Amer. Math. Soc. 93 (1959), 418-491.

[GM99] Oscar Gonzalez and John H. Maddocks, Global curvature, thickness, and the ideal shapes of knots, Proc. Nat. Acad. Sci. (USA) 96 (1999), 4769-4773.

[Kau89] Louis H. Kauffman, Invariants of graphs in three-space, Trans. Amer. Math. Soc. 3 (1989), 697-710.

[KS97] Robert B. Kusner and John M. Sullivan, On distortion and thickness of knots, Topology and Geometry in Polymer Science (S. G. Whittington, D. W. Sumners, and T. Lodge, eds.), IMA Vol. 103, Springer, 1997, pp. 67-78; arXiv.org/dg-ga/9702001.

[Kup94] Greg Kuperberg, Quadrisecants of knots and links, J. Knot Theory Ramifications 3 (1994), 41-50; arXiv.org/math.GT/9712205.

[Mil50] John W. Milnor, On the total curvature of knots, Ann. of Math. 52 (1950), 248-257. 
[Moi54] Edwin E. Moise, Affine structures in 3-manifolds. VIII. Invariance of the knot-types; local tame imbedding, Ann. of Math. 59 (1954), 159-170.

[Sul08] John M. Sullivan, Curves of finite total curvature, Discrete Differential Geometry (A. I. Bobenko, P. Schröder, J. M. Sullivan, G. M. Ziegler, eds.), Oberwolfach Seminars, vol. 38, Birkhäuser, 2008, this volume, to appear; arXiv.org/math.GT/0606007.

[Tan98] Kouki Taniyama, Total curvature of graphs in Euclidean spaces, Differential Geom. Appl. 8:2 (1998), 135-155.

[Thu97] William P. Thurston, Three-dimensional geometry and topology, Princeton, 1997, edited by Silvio Levy.

Elizabeth Denne

Department of Mathematics \& Statistics

Smith College

Northampton, MA 01063

USA

e-mail: edennedemail.smith.edu

John M. Sullivan

Institut für Mathematik, MA 3-2

Technische Universität Berlin

Str. des 17. Juni 136

10623 Berlin

Germany

e-mail: sullivanemath.tu-berlin.de 